\title{
EPIDEMIOLOGIA DA INFECÇÃO POR TOXOPLASMA GONDII NO MUNICÍPIO DE RIBEIRÃO DAS NEVES, MG. I. IMPORTÂNCIA DOS ANIMAIS DOMÉSTICOS COMO FONTE DE INFECÇÃO DO T. GONDII PARA O HOMEM
}

\author{
Maria Cristina Viana de Camargo, Carlos Mauricio de \\ Figueiredo Antunes e Cléa de Andrade Chiari
}

\begin{abstract}
Foi realizado um estudo retrospectivo ou caso controle em uma amostra de 500 pessoas selecionadas aleatoriamente da população de Ribeirão das Neves, $M G$, Brasil, durante o periodo de junho de 1983 a janeiro de 1984, para verificar se variáveis ligadas ao contato com animais no domicilio estariam associadas a ocorrência da infecção bumana por Toxoplasma gondii. Foram obtidas associações estatisticamente significativas com relaçāo ao contato com gatos, galinhas e porcos no domicilio e nenbuma associação quanto ao consumo de carne, leite e ovos.
\end{abstract}

Palauras-chaves: Epidemiologia. Toxoplasmose. Animais domésticos.

A infecção por $T$. gondii pode se disseminar entre os animais e o homem através dos mais variados mecanismos de transmissão como a ingestão de oocistos eliminados junto com as fezes de felídeos, que se espalham pelo meio ambiente contaminando o solo, a água, os alimentos e locais de pastagens ${ }^{19}$; a ingestão de cistos presentes nos tecidos e órgãos de animais infectados ${ }^{13}$; o contato direto com secreções de animais infectados ${ }^{11}$ e mecanismos de transmissão congênita ${ }^{6}$. Além destes, pela ingestão de hospedeiros transportadores ou alimentos por eles contaminados ${ }^{11}$ e por transfusão sanguinea ${ }^{1}$.

Entretanto, apesar dos mecanismos de transmissão da toxoplasmose serem conhecidos alguns aspectos ligados à cadeia de transmissão do parasita ainda não estão claros. Dentre eles, o papel dos animais domésticos como fonte de infecção para o homem continua sendo objeto de discussão.

Alguns autores acreditam que pelo fato dos animais domésticos coabitarem com o homem, passariam a funcionar como fonte de infecção

\footnotetext{
Departamento de Parasitologia, Instituto de Ciências Biológicas, Universidade Federal de Minas Gerais, Belo Horizonte, MG.

Endereço para correspondência: Prof ${ }^{a}$. Maria Cristina Viana de Camargo. Dept ${ }^{\circ}$ de Parasitolologia/ICB/UFMG. Caixa Postal 486, 30161-970 Belo Horizonte, MG, Brasil.

Recebido para publicação em 14/06/94.
}

para este, que se contaminaria através do contato direto com suas secreções e ingestão de carne, leite e ovos contaminados ${ }^{813} 18$

Para outros, o contato com animais domésticos não resulta necessariamente na infecção do homem. Foi demonstrado que a freqüência de anticorpos anti- $T$. gondii entre grupos expostos a contato com animais, inclusive o gato, é a mesma que em outros grupos estudados, não expostos ${ }^{12}$.

Este trabalho teve como ob etivo verificar a importância dos animais criar os no domicílio e o seu papel na transmissão da toxoplasmose humana.

\section{MATERIAL E MÉTODOS}

O trabalho foi realizado no município de Ribeirão das Neves, localizado na zona metalúrgica, fazendo parte da região metropolitana de Belo Horizonte. Sua superfície apresentava $151 \mathrm{~km}^{2}$ de extensão com uma população de 62.278 habitantes (Censo, IBGE, 1980).

Foi realizado um estudo retrospectivo ou caso-controle, em uma subamostra de 500 pessoas selecionadas aleatoriamente da população de Ribeirão das Neves, durante o período de junho de 1983 à janeiro de 1984 . Esta subamostra foi obtida através de um mapeamento da sede do município onde todos os quarteirões foram enumerados. Destes, alguns foram sorteados e realizada 
Camargo MCV, Antunes CMF, Cbiari CA. Epidemiologia da infeç̧âo por Toxoplasma gondii no município de Ribeirão das Neves, MG. I. Importância dos animais domésticos como fonte de infecção do T. gondii para o bomem. Revista da Sociedade Brasileira de Medicina Tropical 28:211-214, jul-set, 1995.

uma visita em todas as casas, preenchendo formulário para cada habitante, obtendo-se uma amostra aleatória de 4.141 habitantes.

$\mathrm{Na}$ amostra foi realizada a coleta de sangue para a realização do diagnóstico da infecção por $T$. gondii através da Reação de Imunofluorescência Indireta (RIFI), Camargo ${ }^{4} \mathrm{e}$ aplicado um questionário visando obter informações sobre variáveis ligadas ao contato do homem com animais na residência em que morou mais tempo no passado e na residência atual.

Amostras de sangue foram retiradas por punção venosa, o soro era separado no mesmo dia da coleta e conservado a $-20^{\circ} \mathrm{C}$ até a realização das reações. Foi utilizado imune soro antigamaglobulina humana, classe IgG, marcada com isotiocianato de fluoresceína, relação fluoresceína/proteína $=9$, produzido no Laboratório de Toxoplasmose/ICB/UFMG.

Como antígeno, foi utilizado taquizoíto de T. gondii, amostra AS-28, mantida em laboratório por passagens sucessivas em camundongos.

$\mathrm{Na}$ construção do questionário foram obtidas informações sobre criação de cães, gatos, galinhas, cabras e porcos avaliando-se o grau de contato com estas espécies, se constante ou esporádico; contato com roedores domésticos e hábitos de consumo de carne, leite e ovos considerando-se a origem do alimento, o modo de preparo e a frequência em que era consumido pela população.

As variáveis foram comparadas entre dois grupos, aqueles que apresentaram RIFI reativa (soros reagentes em diluições iguais ou maiores que $1: 16$, título mínimo significativo) foram considerados os casos e os que apresentaram RIFI não reativa, os controles.

Utilizou-se o "Statistical Package for Social Science" (SPSS). Para estabelecer as associações entre as variáveis estudadas e a ocorrência da infecção por $T$. gondii foram utilizadas para as variáveis contínuas o teste " $\mathrm{t}$ " de significância e para as variáveis discretas ou qualitativas o teste $\mathrm{X}^{23}$

\section{RESULTADOS}

\section{Contato com animais no domicílio}

A proporção de indivíduos que apresentaram contato com gatos no domicílio, tanto no passado, como na residência atual, foi estatisticamente maior no grupo com sorologia reativa do que no grupo com sorologia não reativa (Tabela 1 ).

Tabela 1 - Distribulção da Reação de Imunofluorescência Indireta (RIFI) para T. gondii segundo variäveis associadas ao contato com gatos no domicilio.

\begin{tabular}{|c|c|c|c|c|c|c|c|c|}
\hline \multirow{4}{*}{ Variáveis } & \multicolumn{8}{|c|}{ RIFI } \\
\hline & \multicolumn{4}{|c|}{ Passado } & \multicolumn{4}{|c|}{ Presente } \\
\hline & \multicolumn{2}{|c|}{ reativa } & \multicolumn{2}{|c|}{ nāo reativa } & \multicolumn{2}{|c|}{ reativa } & \multicolumn{2}{|c|}{ não reativa } \\
\hline & $\bar{n}$ & $\%$ & $\mathbf{n}$ & $\%$ & $\overline{\mathrm{n}}$ & $\%$ & $n$ & $\%$ \\
\hline \multicolumn{9}{|l|}{ Contato* } \\
\hline $\operatorname{sim}$ & 142 & 47,8 & 60 & 29,6 & 100 & 33,7 & 35 & 17,2 \\
\hline nāo & 152 & 52,2 & 143 & 70,4 & 197 & 66,3 & 168 & 82,8 \\
\hline Total & 294 & 100,0 & 203 & 100,0 & 297 & 100,0 & 203 & 100,0 \\
\hline
\end{tabular}

Foram excluídas, na apuração, as respostas "não sei" (ns), os casos em que o entrevistado nâo respondeu (nr) e os casos de questāo não aplicável (na).

* Registradas diferenças significativas ao nivel de 0,05 (proporçōes: $\mathrm{X}$ : heterogeneidade)

Da mesma forma, a proporção de indivíduos que apresentaram contato "constante" com galinhas criadas em fundo de quintal, no passado, como na residência atual, foi estatisticamente maior no grupo com sorologia reativa do que no grupo com sorologia não reativa (Tabela 2 ).

Tabela 2 - Distribuição da Reação de Imunofluorescência Indireta (RIFI) para T. gondil segundo variáveis associadas ao grau de contato com galinhas no domicílio.

\begin{tabular}{|c|c|c|c|c|c|c|c|c|}
\hline \multirow{4}{*}{ Variáveis } & \multicolumn{8}{|c|}{ RIFI } \\
\hline & \multicolumn{4}{|c|}{ Passado } & \multicolumn{4}{|c|}{ Presente } \\
\hline & \multicolumn{2}{|c|}{ reativa } & \multicolumn{2}{|c|}{ nảo reativa } & \multicolumn{2}{|c|}{ reativa } & \multicolumn{2}{|c|}{ não reativa } \\
\hline & $\mathrm{n}$ & $\%$ & $n$ & $\%$ & $\bar{n}$ & $\%$ & 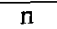 & $\%$ \\
\hline \multicolumn{9}{|l|}{ Grau* } \\
\hline constante & 82 & 44,8 & 34 & 29,3 & 64 & 47,8 & 28 & 31,5 \\
\hline esporádico & 101 & 55,2 & 82 & 70,7 & 70 & 52,2 & 61 & 68,5 \\
\hline Total & 183 & 100,0 & 116 & 100,0 & 134 & 100,0 & 89 & 100,0 \\
\hline
\end{tabular}

Foram excluídas, na apuraçào, as respostas "não sei" (ns), os casos em que o entrevistado não respondeu (nr) e os casos de questão nào aplicável (na).

- Registradas diferenças significativas ao nível de 0,05 (proporções: $\mathrm{X}^{2}$ heterogeneidade).

Com relação ao contato com porcos no domicílio no passado, a proporção encontrada foi estatisticamente maior no grupo de indivíduos com sorologia reativa do que no grupo com sorologia não reativa (Tabela 3 ).

Tabela 3 - Distribuicão da Reação de Imunofluorescência Indireta (RIFI) para $T$. gondit segundo variáveis associadas ao contato com porcos no domicílio, no passado.

\begin{tabular}{|c|c|c|c|c|}
\hline \multirow{3}{*}{ Variáveis } & \multicolumn{4}{|c|}{ RIFI } \\
\hline & \multicolumn{2}{|c|}{ reativa } & \multicolumn{2}{|c|}{ não reativa } \\
\hline & $n$ & $\%$ & $n$ & $\%$ \\
\hline \multicolumn{5}{|l|}{ Contato* } \\
\hline $\operatorname{sim}$ & 137 & 46,1 & 69 & 34,2 \\
\hline não & 160 & 53,3 & 133 & 65,8 \\
\hline Total & 297 & 100,0 & 202 & 100,0 \\
\hline
\end{tabular}

Foram excluídas, na apuração, as respostas "não sei" (ns) os casos em que o entrevistado não respondeu (nr) e os casos de questão não aplicável (na).

* Registradas diferenças significativas ao nível de 0,05 (proporções: $\mathrm{X}^{2}$ heterogeneidade). 
Camargo MCV, Antunes CMF, Chiari CA. Epidemiologia da infecção por Toxoplasma gondii no municîpio de Ribeirão das Neves, MG. I. Importância dos animais domésticos como fonte de infecção do T. gondii para o homem. Revista da Sociedade Brasileira de Medicina Tropical 28:211-214, jul-set, 1995.

Variáveis associadas ao contato com cães, cabras e roedores no domicílio não influenciaram na distribuição da RIFI para $T$. gondii nos indivíduos.

\section{Consumo de carne, leite e ovos}

Variáveis associadas ao consumo de carne de boi, porco, galinha e caça; consumo de ovos de galinhas caipiras ou de granja; consumo de leite de vaca e cabra, cru ou fervido e a frequência em que todos estes produtos eram consumidos pela população não apresentaram associação com a distribuição da RIFI para $T$. gondii entre os indivíduos.

\section{DISCUSSÃO}

O contato com gatos domésticos parece ser fator relevante na transmissão da infecção por T. gondii em Ribeirão das Neves. A associação vem reforçar os dados já existentes na literatura os quais demonstram a importância do papel desempenhado pelos felídeos na transmissão e manutenção do ciclo do $T$. gondii na natureza e através de relatos sobre a ocorrência da toxoplasmose humana e contato com gatos ${ }^{109}$.

O papel de galinhas domésticas como fonte de infeç̧ão do $T$. gondii para o homem é ainda pouco estudado, apesar de ter sido relatado na literatura o encontro de correlações positivas entre contato com aves comestiveis e sorologia reativa para $T$. gondi ${ }^{14}$ ${ }^{15}{ }^{16}$. No município, a relação estabelecida com grau de contato com galinhas domésticas pode estar associada ao aumento da oportunidade de adquirir a infecção através do consumo de carne e/ou ovos parasitados, mas merece ser melhor investigada, principalmente entre as populações de áreas rurais cujo hábito parece ser mais frequente.

As associações encontradas com relação ao contato com porcos no domicílio confirmam o papel destas espécies como bons reservatórios do $T$. gondii apresentando altas taxas de infecção natural ${ }^{16}$. Mas, de acordo com alguns autores, o consumo de carne de animais infectados, só é importante como fónte de infecção, em populações onde exista o hábito de ingerí-la crua ou mal cozida ${ }^{1320}$

$O$ contato com cão doméstico não foi importante na transmissão da toxoplasmose humana em Ribeirão das Neves, apesar da infecção ser comum nestas espécies?. A importância epidemiológica dos cães: na manutenção do nicho antropológico do $T$. gondii ainda não está clara.

Da mesma forma, não houve associação com o contato com cabras no domicílio e o hábito de ingerir seu leite, apesar desta espécie ser um bom reservatório do $T$. gondii, principalmente quando criada em estreita relação com o homem, e o seu leite e carne foram algumas vezes incriminados como fonte de infecção ${ }^{18}$.

Em relação ao papel dos bovinos como fonte de infecção do T. gondii para o homem, os resultados foram os esperados. Tentativas de isolamento do parasita em amostras de carne de boi e vaca tem sido negativas. A persistência do parasitismo nestas espécies é considerada baixa, diminuindo o risco de infecção também através da ingestão de seu leite contaminado ${ }^{17}$.

Os dados encontrados vêm reforçar a importância do confinamento de algumas espécies de animais no domicílio e sua possivel relação com a ocorrência das infecções por Toxoplasma gondii.

\section{SUMMARY}

During a six month period (June 1983 to January 1984), a case-control study was carried out to determine if the variables associated with domestic animals contact would be correlated with the prevalence of Toxoplasma gondii infection. A randomized sample of 500 subjects from the population of Ribeirão das Neves, Minas Gerais, Brazil was studied. A statistically significant correlation was found with cat, chicken and pig contact but none with consumption of meat, milk and eggs.

Key-words: Epidemiology. Toxoplasmosis Domestic animals.

\section{REFERÊNCIAS BIBLIOGRÁFICAS}

1. Amato Neto V, Cotrin JX, Laus WC, Gomes MCO. Nota sobre o encontro de Toxoplasma gondii $\mathrm{em}$ sangue destinado à transfusão. Revista do Instituto de Medicina Tropical de São Paulo 5:6869, 1963.

2. Berquó ES, Souza JMP, Gotlieb SLD. Teste de uma média populacional. In: Berquó ES, Souza JMP, Gotlieb SLD (eds) Bioestatística, $1^{\text {a }}$ edição, Ed. Pedagógica e Universitária Ltda, São Paulo p. 215 222, 1981. 
Camargo MCV, Antunes CMF, Chiari CA. Epidemiologia da infecçẫo por Toxoplasma gondii no município de Ribeirão das Neves, MG. I. Importância dos animais domésticos como fonte de infecção do T. gondii para o bomem. Revista da Sociedade Brasileira de Medicina Tropical 28:211-214, jul-set, 1995.

3. Berquó ES, Souza JMP, Gotlieb SLD. Teste de uma variância populacional. In : Berquó ES, Souza JMP, Gotlieb SLD (eds) Bioestatística, $1^{a}$ edição, Ed. Pedagógica Ltda, São Paulo p. 247-248, 1981.

4. Camargo ME. Improved technique of indirect immunofluorescence for serological diagnosis of toxoplasmosis. Revista do Instituto de Medicina Tropical de São Paulo 6:117-118, 1964.

5. Chiari CA, Neves DP. Human toxoplasmosis acquired by goat's milk ingestion. Memórias do Instituto Oswaldo Cruz 79:337-340, 1984.

6. Desmonts G, Couvreur J. Toxoplasmosis in pregnancy and its transmission to the fetus. Bulletin of the New York Academy of Medicine 50:146-159, 1974.

7. Ferraroni JJ, Marzochi MCA. Prevalência da infecção pelo Toxoplasma gondii em animais domésticos, silvestres e grupamentos humanos da Amazônia. Memórias do Instituto Oswaildo Cruz 75:99-109, 1980.

8. Frenkel JK, Ruiz A. Human toxoplasmosis and cat contact in Costa Rica. The American Journal of Tropical Medicine and Hygiene 29:1167-1180, 1980.

9. Frenkel JK, Ruiz A. Endemicity of toxoplasmosis in Costa Rica.American Journal of Epidemiology 113:254-269, 1981.

10. Frenkel JK, Ruiz A, Chinchilla M. Soil survival of Toxoplasma oocysts in Kansas and Costa Rica. The American Journal of tropical Medicine and Hygiene 24:439-443, 1975.

11. Galuzo IG. The epizootiology of toxoplasmosis. In: Fitzgerald PR (ed) Toxo plasmosis of animials, 1st edition, Urbana, College of Veterinary Medicine and Agricultural Experiment Station University of Illinois p. 395-413, 1970.

12. Ganley JP, Constock GW. Association of cats and toxoplasmosis. American Journal of Epidemiology 111:238-246, 1980.
13. Jacobs L, Melton ML.A procedure for testing meat samples for Toxoplasma with preliminary results of a survey of pork samples. Journal of Parasitology 43(suppl):38-39, 1957.

14. Jones FE, Melton ML, Lunde MN, Eyles DE, Jacobs L. Experimental toxoplas mosis in chickens. Journal of Parasitology 45:31-37, 1959.

15. Kimball AC, Bauer H, Sheppard CG, Held JR, Shuwan LM. Studies on toxoplas mosis. III. Toxoplasma antibodies in obstetrical patients correlated with residence, animal contact and consumption of selected foods. American Journal of Hygiene 71:93-119, 1959.

16. McCulloch WE, Braun JL, Heggen DW. Studies on medical and veterinary stu dents skin tested for toxoplasmosis. Public Health Reports (Wash) 78:689-698, 1963.

17. Passos LMF. Determinação da infecção por Toxoplasma gondii em bovinos e suínos abatidos em Belo Horizonte (MG) através da frequência de anticorpos e tentativa de isolamento à partir de músculos de bovinos. Tese de Mestrado, Universidade Federal de Minas Gerais, Belo Horizonte, MG, 1984.

18. Riemann HP, Meyer ME, Theis JH, Kelso G, Behymer DE. Toxoplasmosis in an infant fed unpasteurized goat milk. Journal of Pediatrics 87:573576, 1975.

19. Ruiz A, Frenkel JK. Isolation of Toxoplasma from cat feces deposited in false attics of homes in Costa Rica. Journal of Parasitology 63:931-932, 1977.

20. Schenk MAM. Freqüência e isolamento de Toxoplasma gondii em suínos do Estado de Minas Gerais. Tese de Mestrado, Universidade Federal de Minas Gerais, Belo Horizonte, MG, 1976. 